\title{
Malraux-Israël: retours à la Genèse
}

\author{
Sergio Villani \\ Université York
}

N orthrop Frye affirme que les structures de l'imagination occidentale, de sa littérature, dérivent presque entièrement de la Bible, et en particulier de l'Ancien Testament. ${ }^{1}$ Ce principe informe cette étude sur Malraux et le lieu d'Israël. Plus qu'un espace géographique, plus qu'une entité politique, Israël représente pour Malraux un espace biblique et litteraire où l'on peut lire et retrouver les traces de l'évolution spirituelle et culturelle de l'humanité. Israël sera pour lui un point cardinal de son imagination, de sa méditation, où le penseur et l'écrivain séjournent souvent dans leur penchant, dans cette obsession même, de remonter aux origines afin de découvrir ce qui est fondamental, essentiel, dans la nature de l'homme et de l'art. D'ailleurs, pour cet intellectuel qui nourrit un culte de figures héroïques, comme Lawrence, comme Saint-Just, Israël va incarner pour lui un personnage, majeur, monumental, comme eux à la fois mythique et héroïque, de son panthéon. A travers cette image, il nous sera possible de comprendre, et d'expliquer peut-être, cette métamorphose politique et littéraire, cette volte-face intrigante, ${ }^{2}$ que fait ou subit Malraux au cours des années quarante. Enfin, penser Israël, ce sera pour Malraux encore une occasion d'exercer son goût pour le meditatio et l' imitatio, cette nécessité de passer de la méditation à l'action, de l'action à la méditation, et donc satisfaire à la fois son besoin intellectuel et son engagement social.

Il est vrai que Malraux, ce voyageur obsédé, infatigable, cet écrivain pour qui le voyage, le picaresque on pourrait dire, est presque une précondition de son écriture, n'a jamais visité le pays d'Israël. Il l'a contourné, pour ainsi dire, visitant le Yémen, l'Egypte, la Grèce, restant loin de cette focalisation à haute fréquence de passions spirituelles et politiques. Pourtant, il est aussi vrai que, pendant la plupart de sa vie, Israël n'existait que comme une aspiration nationale. Et lorsque ce rêve devint enfin réalité, visiter ce pays aurait été sûrement indiplomatique, sinon dangereux, pour l'homme d'État et le Ministre d'une France amie incontestable d'Israël mais qui tenait à ne pas aliéner les pays arabes. Il y a, cependant, aussi des considérations d'ordre psychologiques et spirituelles qui pourraient expliquer au moins en partie cette absence. Voulait-il d'une manière volontaire ou involontaire, s'écarter de la possibilité d'un bouleversement émotionnel-spirituel qui serait provoqué par une visite à cette terre sacrée? Et même s'il avait trouvé le courage de le faire, oser le faire, et s'il avait réussi à rester impassible devant le poid spirituel de ces lieux, n'aurait-on peut-être mal interprêté sa visite?

Ce qui demeure incontournable, c'est sa fraternité pour les enfants d'Israël. Cette affection se manifeste dans sa lutte acharnée contre le fascisme et contre toute autre forme de racisme criminel. On la retrouve dans sa condamnation absolue des camps d'extermination et de toute autre violence dirigée contre l'humiliation et la destruction de 
l'être humain. D'une manière plus tangible et directe, cette amitié se reconnaît dans son respect et son admiration d'artistes tels Serge Eisenstein, Max Jacob et Marc Chagall. Avec celui-ci, il participe en 1955 à rendre hommage à la terre et au peuple d'Israël. Sa préface aux livre photographique d'Izis, ${ }^{3}$ demeure un témoignage fort et sincère de ce peuple chez qui il voit exemplifiés ce courage et cette détermination de s'affirmer contre toute son histoire de soumission, de persécution, de dispersion et d'exil. Malraux voit surgir dans ces photos, grâce à cet art qui "saisit l'instant où se reflètent des siècles, l'instant qui métamorphose le réel en le prolongeant dans l'interrogation du poème" (p.7), ce qu'il appelle une "épopée moderne" et "la trouble majesté d'une obsession spirituelle." C'est ainsi que, d'une perspective millénaire, sa méditation sur l'histoire du peuple hébreu relève une double orientation: d'un côté, la sagesse qui prêche la soumission pour assurer la survivance et la permanence, -- croire que "la dernière victoire" ne dépend que de Dieu (p. 10); de l'autre côté, il y a des images de révolte, de résistance, de cette volonté du héros qui s'oppose au sacré, qui prend des armes pour s'affirmer et mettre fin à "l'indomptable soumission." Malraux exprimera de nouveaux cette double postulation, -- ce tiraillement, et enfin harmonie, entre la soumission et la résistance du peuple d'Israël tout le long de son histoire, en 1960 dans son allocution prononcée à l'occasion du "Centenaire de l'Alliance israélite universelle." ${ }^{4}$ L'antécédent de ce discours, le texte-préface de Malraux au livre de photos d'Izis, s'ouvre et se clôt avec cette antimonie existentielle et spirituelle. Au début comme à la fin de ce texte, Malraux attire notre attention sur l'image de la sentinelle qui veille sur les collines de Jérusalem, dominant à la fois et l'espace et les temps. Au début, cette image résolue se dresse contre la voix prophétique d'Isaïe: "le fusil de la sentinelle israélienne qui semble garder la frontière contre les Arabes, contre le crépuscule et contre l'Eternel" (p. 7); à la fin, par la voie d'images poétiques, cette opposition atteint des dimensions héroïques et millénaires: "et dans l'ombre de Schéol qui s'approfondit tandis que montent les étoiles du Déluge, les Juges d'Israël regardent le petit veilleur condamné contre lequel ne prévaut pas la menace divine, parce que le mystère de la plus humble grandeur n'est pas moins profonde que celui de la mort" (p.11). Dans ces deux moments, début et fin de ce texte, scandé par la répétition de la préposition "contre," Malraux évoque l'image archaïque, originelle, imposante de ce révolté prométhéen, celle du premier Israël, celle de Jacob qui sur une montagne lutta toute une nuit avec l'Ange, avec l'Eternel, et se retrouva au petit matin avec une nouvelle identité et une nouvelle mission. Nous allons revenir sur cette image de "la lutte avec l'ange" et la lumière qu'elle jette sur le combat de Malraux luimême. Comme dans le cas de Jacob, ${ }^{5}$ cette métaphore représente une métamorphose et l'engagement pour son peuple qui en résulte.

En lisant cette préface, on rencontre un esprit qui approfondit une vision globale et intemporelle. On comprend que, malgré son absence de l'état d'Israël, surtout à travers ses écrits, Malraux est fréquemment le visiteurs de cet espace biblique et légendaire, actuel et immémorial. On y perçoit la largeur et la profondeur de ses connaissances dans ce domaine et on s'émerveille de l'ampleur poétique qu'il confêre à ces considérations. Il fait

\section{LittéRéalité 48}


résonner de nouveau cette poésie dont les échos remontent à la poésie biblique de la Genèse, à la fin de son discours en 1960 à l'Unesco:

Beaucoup d'entre vous ont contemplé les veilleurs des murailles, à l'heure où le soleil se couche derrière les collines qui ont vu dix-sept fois détruire Jérusalem. Ils veillent sur les filles qui rêvent à la chanson de la nuit, comme au temps du Cantiques des Cantiques: Mon bien-aimé vient le samedi -- Ma mère sommeille, mon père dort --Seuls mon coeur et moi sommes éveillés...; et dans l'ombrequi s'approfondittandis que montent les étoiles qui virent le Déluge, les guetteurs semblent étendre jusqu'à elles le chant funèbre des morts guerriers sur le plateau d'argent. ${ }^{6}$

Israël, lieu de la mémoire, est donc surtout pour Malraux un espace de limaginaire, un riche domaine littéraire d'où il puise des images, des structures, et des figures hérol̈ques qu'il intègre dans ses récits et qui l'aident à mieux comprendre, à mieux expliquer et, parfois, à survivre certains moments déterminants de sa vie. Cette remontée méditative vers la Genèse, comme vers une source, dévoile sa démarche intellectuelle, de retourner au commencement, à l'origine, au primordial, afin d'y découvrir l'essentiel et y découvrir aussi comment cette essence se transmet dans le temps. C'est ainsi qu'il élucide et élabore le principe de la métamorphose, en se déplaçant dans le temps, vers un passé de formes primaires. Par ce moyen, il s'attendait à formuler une psycholgie de l'art; il aboutit à en formuler une métaphysique.

Le procédé de ces retours en arrière consiste d'un graduel dépouillement, ou dénudement, des vestiges de temps et de civilisation. On revient ainsi à un état primaire, de formes simples dans lesquelles on peut lire une sagesse, une connaissance existentielle brute, amère, parfois tragique, parfois effrayante, qui dépasse l'espace et le temps. En fait, le Ministre de la Culture, renommé pour son programme de décrassement de monuments et de bâtiments, n'inaugurait rien de nouveau dans ce domaine. Dans ces livres, l'écrivain avait déjà pratiqué, depuis les années vingt, une sorte de décrassement de la forme humaine. L'aventure dans les forêts vierges de l'Indochine décrit déjà un retour à un temps immémorial. Dans La Voie royale, à mesure qu'on pénètre "au coeur des ténébres," comme dans le roman de Conrad, on s'approche d'une forme de l'humain on ne peut plus réductible. La marche à travers la forêt humide, palpitante, collante, grouillante d'insectes, dans un monde prédéluvien,

[...] décomposition de l'esprit dans cette lumière d'aquarium [...] Ils avaient rencontrés déjà des petits monuments écrasés, aux pierres si serrés par les racines qui les fixaient au sol comme des pattes qu'ils ne semblaient plus avoir été élevés par des hommes mais par des êtres disparus habitués à cette vie sans horizon, à ces ténèbres marines. [...] dérivé du dégoût par le 
danger, il retomba sur le mur avec une brutalité de bête en fuite, avançant de nouveau, ses mains gluantes collées aux feuilles pourries, hébété de dégoût [...] Il s'arrêta, stupide: dans cette position, il ne savait plus sauter. [...] Libre enfin de courir comme un homme, il se jeta en avant $[. . .]^{7}$

trace une pente vers le primitif: ce retour au primitif sert de prémonition à la descente vers l'inhumain, à la rencontre avec Grabot. Aveugle, attelé, tournant en rond autor d'une meule massive, Grabot figure l'abrutissement total de l'être humain. Les bruits quil émet traduisent la souffrance et la misère de tous les âges; son silence humilié parie de l'ultime dégradation. Nous y confrontons le Mal que peut faire l'homme contre l'homme, la pire des humiliations. Aucun Sysiphe ne pourrait symboliser un état si tragique, si loin et pourtant si proche, un état tellement humain. On éprouve de la pitié; on ressent la peur, car on reconnaît dans le visage de Grabot celui de tout le monde. Cette technique donc permet de creuser dans le temps et dans l'espace jusqu'à ce que temps et espace se croisent et disparaissent dans une figure primordiale qui universalise une vérité humaine indéniable. Quelques années plus tard, l'aventure, "aventure géographique," à la recherche de Saba, invite à un voyage au fond du temps, dans les terres légendaires de la Genèse. Saba devient l'image de cette hantise humaine de forer dans le temps à la recherche d'une identité originelle. Cette fois on pénètre une géographie au contrepoint de la forêt luxueuse décrite dans La Voie royale, dans une "forêt " (p. 114) qui flambe, une lande aride, "stérile," rocheuse, mais pays aussi inhabité d'animaux rampants et millénaires, les lézards (p. 81), les scorpions (p.114) et "la mouche, reine de ces solitudes" (p. 83). Ici, au lieu des ruines du temple enfoui dans les feuillages, on va à la rencontre des ruines du "prodigieux spectacle de la cité morte" (p.75), "ce naufrage échoué là comme un vaisseau babylonien plein de statues brisées" (p. 82). Ici, dans le désert yéménite, au lieu du visage abruti de Grabot, on rencontre la figure auguste de Salomon, le poète, l' amoureux, le prophète, paragon de la sagesse même. Saba, la reine, elle demeure, en filigrane, la beauté légendaire, le rêve, l'idéal qui attire le roi vers ces terres désertiques. Au centre du récit s'élève la statuesque image de Salomon, "le Roi qui avait écrit le plus grand poème du désespoir" (p. 107), ici le représentant de tous les "farfelus", aventuriers qui partent, sacrifiant tout, à la recherche d'un rêve. Le Roi figé en statue, levant le bras à travers les sables dans la direction du palais de la reine de Saba, est le le symbole du résistant, de la sentinelle qui veille, comme celle dans le livre d'Izis, pour protéger la beauté, l'amour, Saba, contre les "démons," les ravages du temps. Comme la femme de Lot qui ne peut s'empêcher de se tourner vers le passé, il est réduit en poussière, comme tout dans cette Vallée des Tombeaux, d'une manière dérisoire: par l'oeuvre d'un insecte foreur:

Depuis des annees, Salomon avait fui Jérusalem. Asservis au sceau dont le dernier caractère ne peut être lu que par les morts, ses démons l'avaient suivi à travers le désert. Et dans une vallée de Saba, le Roi qui avait écrit

\section{LittéRéalité \$ 50}


le plus grand poème du désespoir regardait, mains croisées sous le menton et appuyées sur le haut baton de voyage, les démons qui depuis tant d'années élevaient le palais de la Reine. Il ne bougeait plus jamais, montrant seulement de l'index le sceau tout-puissant. Comme l'ombre des soldats romains à demi ensevelis, son ombre chaque soir s'étendait jusqu'aux confins du désert, et les démons du sable travaillaient toujours, envieux de leurs frères libres qui hurlaient à travers le désert avec la voix des tombes. Un insecte vint, qui cherchait du bois. Il vit le bâton royal, attendit, prit confiance, commença de le forer. Bâton et roi tombèrent en poussière: le Seigneur du silence avait voulu mourir debout pour asservir à jamais à la Reine tous les démons qu'il gouvernait. Délivrés, ils coururent à la ville. Elle était déjà en ruine, et la Reine était morte depuis trois cents ans (p. 107-108).

Ce conte de la folie du Roi, symbole de la sagesse, comporte des aspects du conte philosophique et sert donc de leçon existentielle: ici, la vanité de fixer les yeux vers le passé et de vouloir, comme dit Valéry, entrer dans l'avenir à rebours. ${ }^{9}$ Malraux, lui aussi étant parmi les "farfelus", les aventuriers dont il parle, se regarde dans son image du Roi Salomon comme dans un miroir et reconnaît sa propre folie. Cette conscience va l'inciter à s'engager dorénavant davantage dans le présent, dans l'actuel de sa société occidentale, et cultiver une vision tournée vers l'avenir, surtout en luttant contre le fascisme et en épousant davantage la discipline d'une éthique de gauche.

Ce sera une autre géographie, cette fois-ci la terre ocre d'Espagne, qui va engager ses énergies et stimuler son imagination. Au cours de ces années, il trouvera nécessaire encore une fois de revenir à la Genèse pour y tirer des images qui donnent une forme universelle et intemporelle aux réalités de son temps. Dans $L^{\prime} E s p o i r$, au coeur du roman, comme limage de Salomon au centre des textes de Saba, se dessine un lieu infernal, une géhenne, où une "fournaise" flambante permet de voir la souffrance et le désarroi humain à travers les ténébres d'un Schéol, tel celui qui entoure la sentinelle dans le livre d'images d'Izis. Ce lieu infernal fait de noir, de feu et de cris, symbolise un monde désarticulé, fragmenté, tombé en ruines sous la rage de passions déchaînées et de volontés qui s'opposent et s'entrechoquent. La Guerre civile dépossède, dénude, ramène l'être humain à sa forme la plus fondamentale et primaire, à un état de souffrance où la parole même disparaît et devient cri, "jappement." Ironiquement c'est de ce tragique qui jaillit la communion de fraternité. Ce tragique et ce fraternel sont exemplifiés dans la condition du jeune soldat blessé qui hurle dans "l'aquarium" de l'hôpital San Carlos. Ce monde soumarin, résidence d'une humanité fantasmagorique, pansée, estropiée, amputée, compose un autre espace infernal, un lieu sans salut, qui évoque encore une image de déchéance biblique, celle figurée par Jonah. 
Cependant dans L'Espoir, c'est l'image de l'exode qui relie le plus l'imaginaire de Malraux aux formes de la Genèse. La dépossession trouve dans cette image des dimension trans-temporelles. Le tragique dépasse l'individuel et l'immédiat pour aquérir l'ampleur noble de l'épopée. Que ce soit l'exode sur la route entre Malaga et Almeria,

Entre eux la masse passive des sans-logis continuait vers Almeria sa migration désespérée. [...] La route s'engageait sous un tunnel. [...] un campement de grande migration était installé là dans la vie souterraine [...] Un peuple d'ombres s'agitait autour des torches [...] et le bruit des charrettes grondait sous la roche comme un fleuve souterrain, dans un silence si fort qu'il avait gagné jusqu'aux animaux. [...] De nouveau, au-dessus de la coulée du peuple en fuite [...]. ${ }^{10}$

ou celui sur les routes qui sortent de Tolède, misère, souffrance, solitude, mort, amour, pitié, terreur, toute la complexité de l'existence humaine est résumé dans l'image de cette procession infinie d'hommes, de femmes, d'animaux, de charrettes sur une route en fuite, poursuivis par les rafales meurtrières de l'Ange noir, le général Franco. La lutte des résistants et de Malraux est avec et contre ce nouvel ange infernal, une autre incarnation du fascisme..

Malraux reprend cette image de l'exode pour exprimer la dépossession et le déracinement dans le texte "Camp de Chartres," mémorial de son expérience de prisonnier à Chartres qui sert d'ouverture aux Noyers de l'Altenburg, son dernier roman. Le prisonnier y décrit la misère absolue de ses compagnons, abandonnés, isolés, mais qui s'assemblent de temps en temps comme des feuilles mortes emportés par le vent. Ces hommes ont eté dépouillés de tout et réintégrés à un état primitif: "Dans notre tanière engourdie sous le grand soleil de toujours murmure une voix préhistorique" (p. 628). ${ }^{11}$ Ces prisonniers ont été privés de leur humanité. Ils ont perdu leur consistence humaine et, comme les blessés dans l'aquarium de l'hôpital San Carlos dans L'Espoir, ils ne sont que des ombres: "Chaque matin je regarde des milliers d'ombres dans la clarté de l'aube; et je pense: 'C'est l'homme' " (p. 628). Après avor effectué ce retour au primitif, Malraux revient à la Genèse pour approfondir davantage l'image de cette conditon tragique. Il évoque, sur la route au-delà des barbelés, la vision de compatriotes dans un exode de retour vers la capitale, Paris, ce qui fait croire aux milliers de prisonniers à la fin des hostilités: "Sur la route nationale, les autos et les grandes charrettes paysannes maintenant montent vers Paris: le premier reflux de l'exode" (p. 624) Cet espoir, cependant, est vite dégonflé d'une manière dérisoire, car la "manne" qui leur tombe des cieux, ne confirme que leur état d'impuissance irrémédiable. Les feuillets qui tombent sur leur tête n'annoncent pas la paix espéré; ce ne sont que les morceaux de leurs propres lettres, déchirées par les gardes et confiées aux vents.

Enfin l'exode comme motif d'une marche solennelle, commencement et fin

\section{LittéRéalité 52}


humiliant et tragique, est repris par Malraux dans le fragment des Noyers, " De Sens à Chartres". En retraçant les péripéties de ce qu'il nomme "notre débâcle," (p. 797), les événnements militaires qui l'amènent avec un "tas d'esclaves" (p. 790), prisonnier au Camp de Chartres, Malraux amplifie le tragique de la défaite à un niveau national en évoquant l'exode des foules qui fuient dans toutes directions devant l'avancée ou la retraite des armées:

Parallelement a notre marche [...] en silhouette d'un bout à l'autre du ciel, l'exode passe. [...] Ce n'est pas, cette fois, ni la grande migration polonaise, ni le [lacune] des Espagnoles au mouchoir noir, la procession des veuves; mais cet exode-ci rejoint les deux autres, et ceux de la Chine et de la Russie, comme si depuis vingt-cinq ans le cheminement du désespoir continuait autour de la terre. [...] et leur fleuve à peine visible dans le soir coule au pied confus des hautes figures et des chiens allongés qui se découpent làhaut sur la dernière clarté. En Pologne, même en Espagne, ces paysans en fuite (mais comment appeler fuite cette marche funèbre?) c'étaient les gens de l'autre race, celle de la terre. Ceux qui passent devant nous ce soir, je les connais: c'est dans cet exode ou dans un exode pareil [...] La moisson de misère passe sur le soir qui tombe, passe, passe, avec la sombre patience des misérables (p. 770-771).

En faisant allusion aux foules dépossédées au-delà des frontières françaises, en Pologne, en Espagne, en Russie, en Chine, l'amplification de la tragédie atteint des proportions universelles épiques. D'ailleurs, la métaphore du fleuve, aussi bien que celle de la "marche funèbre" et du "cheminement du désespoir," confere une signification existentielle à ce piétinement inifini qui devient la condition errante des "misérables" (p. 771) de tous les lieux et de tous les temps. Enfin, la répétition du mot "exode" relie ces images de la misère actuelle à l'errance du peuple hébreux dans la Genèse, et donc à une destinée de l'humanité intemporelle.

Cet exode de la Genèse est évoqué aussi dans le récit du grand-père, soldat sur le front Russe, qui traverse un terrain infernal, à travers la destruction, la moisson meurtrière faite par l' Ange invisible, le gaz. Sa marche dans la forêt, "dans le dégout" (p. 729), est comme l'avancée dans La Voie royale, une plongée dans l' "horreur" (p.734), pour arriver à une confrontation avec l' inhumain (p.735). Il croit, pendant un moment, retrouver "cette vallée de Terre Promise" (p.735), mais il est bientôt engagé de nouveau à "lutter contre l'inhumain," (p. 738), contre les ravages de "l' Esprit du Mal" (p. 736).

Les allusions ici à l'exode et la métaphore d'une lutte morale, spirituelle dans une géhenne, contre L'Ange noir, contre l'inhumain, opère un dépaysement, un retour à la Genèse, au commencemet. Ce langage métaphorique rappelle aussi la notice de Malraux au début du roman où il affirme que la suite de La Lutte avec l'ange avait été détruite par la

Villani 53 
Gestapo, les Anges noirs du nazisme. Cette notice n'est pas vraiment une explication, mais surtout un prétexte, une technique littéraire pour donner un contexte existentiel et intemporel --une dimension moraliste aussi -- aux faits racontés dans son roman. ${ }^{12}$ D'ailleurs, en évoquant à travers ce titre l'aventure de Jacob dans la Genèse, il se place à côté de ce patriarche, en admirateur de se résistant prométhéen. Après sa lutte avec l'ange, Jacob subit une conversion et une métamorphose. Il acquiert une nouvelle identité, un nouveau nom: il devient Israël, serviteur d'un peuple et d'une nation naissante, avec une mission qui l'arrache à sa vie d' individu égoïste. Une lutte semblable est présentée dans le fragment "De la Vistule à la résistance" où le héros, ayant connu le "conflit de l'homme et le Mal absolu" (p. 813), arrive en France et s'engage dans une cause nationale, dans "la sinistre lutte clandestine" (p. 815) de la Résistance.

Ces épisodes de transformation, de lutte et de métamorphose ne sont-ils pas une tentative de Malraux de donner une forme intemporelle et éthique à sa propre métamorphose? Après la débacle de la France et sa lutte acharnée avec l'Ange, l'Esprit du Mal moderne, comme Jacob il en sort avec une nouvelle identité: il devient comme lui ce "berger" qui sert de guide à son peuple. Il "épouse" la France, dit-il, comme Jacob Israël. Comme Jacob, il renonce à son passé et s'engage à lutter pour un avenir national, reconnaissant la folie de Salomon de vouloir protéger Saba, c'est-à-dire un passé déjà en ruines, déjà tombé en poussière.

Dans le texte qui clôt Les Noyers, titré, comme l'ouverture, "Camp de Chartres," cette re-naissance est figuree par ce que Malraux appelle "la louche poésie des plus vieux contes" (p. 764). Les souvenirs du prisonnier de Chartres font revivre un moment privilegié dans la vie du tankeur, son expérience du "retour sur la terre," du retour à la vie, dans l'émerveillemnet et "la découverte d'un secret simple et sacré" (p.767). A la lumière de l'aube, son sentiment d'ouverture vers l'avenir, de révélation, est lié à l'ouverture apportée aux misérables par l'Enfant de Bethlehem. Cette ouverture est une promesse de liberté pour le prisonnier de Chartres, une promesse que les portes des camps s'ouvriront un jour. Ce sentiment d'ouverture représente l'espoir des hommes, le fondamental, ce qui se transmet à travers les âges. On retrouve souvent l'expression de cette continuité dans les dernières pages des Noyers: dans la retour à la vie après l'enfer semé par le gaz sur le front russe, dans l'émerveillement éprouvé sur les champs de Flandre, dans l'apparition miraculeuse de l'Enfant de Bethlehem, dans la promesse faite à Jacob, et dans le retour au vrai commencement, à l'aube de l'humanité, en rappelant l'admiration de Dieu devant sa création. En fait, la dernière phrase des Noyers de l'Altemburg, opère encore une fois un retour à la Genèse, pour nous faire contempler la forme auguste du premier homme: "Ainsi, peut-être, Dieu regarda le premier homme..." (p. 767). A travers ces retours à l'origine, à la Genèse, Malraux relie le présent à l'intemporel pour affirmer un acte de foi humaniste mais

\section{LittéRéalité \$ 54}


aussi, peut-être, une profession de foi.

\section{Notes}

${ }^{1}$ Northrop Frye, La Parole souveraine: la Bible et la Littérature. Traduit de l=anglais par Catherine Malamoud (Paris: Seuil 1994).

${ }^{2}$ À ce sujet, voir Geoffrey T. Harris, André Malraux, A Reassessment (London: MacMillan Press, 1996; New York: St, Martin's Press, 1996).

${ }^{3}$ Israël, photos d'Izis, préface d'André Malraux, couverture et frontispice de Chagall (Lausanne: La Guilde du Livre, 1955). Voir sur cette préface, Jeanyves Guérin, "Malraux and Israel: Notes on a little known text," Mélanges Malraux Miscellany Vol. XI, no. 1 (Spring 1979): 16-21.

4 "Centenaire de l'Alliance israélite universelle," in André Malraux, Oraisons funèbres (Paris: Gallimard, 1971): 61-74.

${ }^{5}$ Genèse 32:22.

${ }^{6}$ Op. cit: $: 73-74$.

${ }^{7}$ André Malraux, La Voie royale, Oeuvres complètes I (Paris: Editions Gallimard, Pléiade, 1989): $416,420$.

${ }^{8}$ Andre Malraux, La Reine de Saba une "aventure géographique" (Paris: Editions Gallimard, 1993). Les citations sont tirees de cette édition.

${ }^{9}$ Pour d'autres perspectives, voir Waiter Langlois, " The Antimémoires, Saba and the Immortality of Man" in Via Malraux écrits de/ essays by Walter Langlois, réunis par David Bevan (The Malraux Society, 1986): 282-293.

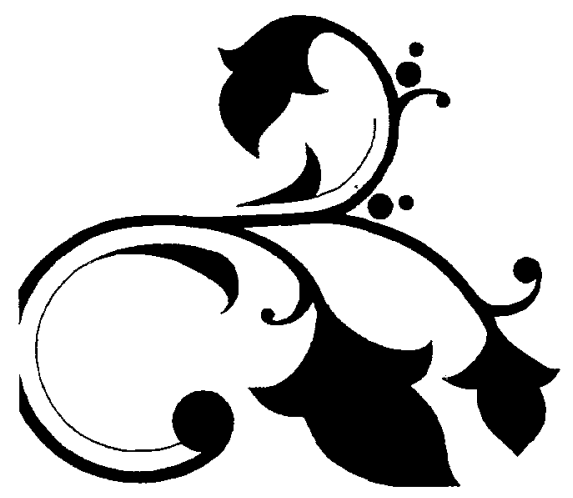

